\title{
HACIA LA PROTECCIÓN SOCIAL UNIVERSAL en AmÉrica Latina. Una CONTRIBUCión
}

AL DEBATE ACTUAL

\author{
Luis Beccaria y Roxana Maurizio*
}

Fecha de recepción: 04 de mayo de 2013. Fecha de aceptación: 18 de septiembre de 2013.

\section{RESUMEN}

El objetivo del documento es contribuir al debate sobre los avances hacia la universalización de la protección social en América Latina. Se aboga a favor de una estrategia que privilegie políticas orientadas a lograr el pleno empleo formal y a consolidar esquemas de regulaciones laborales protectoras pero que se articulen con otras políticas -especialmente sociales- para que, en conjunto, aseguren el acceso universal a servicios sociales básicos de calidad homogénea así como a niveles de ingresos adecuados a lo largo del ciclo de vida.

Palabras clave: América Latina, protección social, política social, servicios básicos de calidad, niveles de ingresos.

Clasificación JEL: E24, H55, I31, O17, D31.

\section{Prospects for Universal Social Protection in Latin America: A Contribution to the Current Debate}

\begin{abstract}
This work aims to contribute to the debate on progress towards achieving universal social protection in Latin America. It advocates a strategy emphasizing policies oriented towards obtaining full formal employment and consolidating protective labor regulations in conjunction with other policies, especially in the social realm, so that together, these ensure universal access to basic social services of the same quality for everyone and adequate income throughout the entire life cycle.
\end{abstract}

Key Words: Latin America, social protection, social policy, quality basic services, income levels

\footnotetext{
*Investigadores de la Universidad Nacional General Sarmiento, Argentina, lbeccari@ungs.edu.ar y rmaurizi@ ungs.edu.ar, respectivamente.
} 
VERS LA PROTECTION SOCIALE UNIVERSELLE EN AMÉRIQUE LATINE. UNE CONTRIBUTION AU DÉBAT ACTUEL.

\section{Résumé}

L'objectif du document est de contribuer au débat sur les avancées vers l'universalisation de la protection sociale en Amérique latine. Il est plaidé en faveur d'une stratégie privilégiant des politiques orientées vers le plein emploi déclaré et la consolidation de schémas de régulation du travail qui protègent les travailleurs mais qui soient articulées avec d'autres politiques -notamment des politiques sociales- pour que, conjointement, elles assurent l'accès universel à des services sociaux de base de qualité homogène ainsi qu’à des revenus appropriés tout au long du cycle de la vie.

Mots clés: Amérique latine, protection sociale, politique sociale, services de base de qualité, niveaux de revenu.

\section{RUMO À PROTEÇÁO SOCIAL UNIVERSAL NA AMÉRICA LATINA. UMA CONTRI- BUIÇÃO AO DEBATE ATUAL \\ Resumo}

O objetivo do documento é contribui ao debate sobre os avanços rumo à universalização da proteção social na América Latina. $\mathrm{O}$ trabalho defende uma estratégia que privilegie políticas orientadas para lograr o pleno emprego formal e para consolidar esquemas de regulaçóes laborais protetoras, mas que se articulem com outras políticas - especialmente sociais - para que, no conjunto, assegurem o aceso universal a serviços sociais básicos de qualidade homogênea, assim como níveis de renda adequados ao longo do ciclo de vida.

Palavras-chave: América Latina, proteção social, política social, serviços básicos de qualidade, níveis de renda.

\section{拉美社会保护普遍化前景：对当前争论的一个贡献}

摘要

本文的目标是对当前拉美实现社会保护普遍化的讨论有所贡献。本文倡导 一种以实现完全正规就业和加强保护性劳工立法为导向的社会政策战略, 且与其他政策在社会领域里相配合。因此, 这能够确保每个人在整个生命 周期中能够享有相同质量的基本公共服务。

关键词：拉丁美洲 社会保护 社会政策 基本服务 收入水平 


\section{INTRODUCCIÓN}

El objetivo de este artículo es contribuir a la reflexión acerca de los enfoques de políticas destinadas a garantizar los derechos económicos y sociales básicos a los ciudadanos en América Latina. El acceso efectivo a, servicios de educación y salud de calidad, a condiciones de trabajo apropiadas y a ingresos monetarios entre otros, a lo largo del ciclo de vida que aseguren niveles adecuados de consumo, es una obligación ética -aun cuando no esté incorporada en compromisos políticos y constitucionales- de los estados. En una sociedad con pleno empleo formal, la mayoría de estos derechos se podría alcanzar por medio de una combinación de mecanismos de seguridad social y normas laborales protectoras. Sin embargo, el pleno empleo formal no ha resultado ser la situación "normal" de las economías capitalistas, incluso las desarrolladas. Más aún, éste no resulta incompatible con marcadas brechas entre los ingresos de distintos grupos de trabajadores, lo que puede traducirse en diferencias en el tipo de bienes y servicios a los que efectivamente acceden, realidad que además tiende a reproducirse intergeneracionalmente. Para atender tales situaciones los estados de las economías avanzadas han desplegado tradicionalmente regímenes de provisión de bienestar con algunos componentes no contributivos buscando, entonces, que el logro de esos derechos no dependa exclusivamente de la situación laboral de los ciudadanos.

Este esquema de protección, en el cual las medidas universales resultan complementos del núcleo constituido por la seguridad social, ha estado sujeto a diversos cuestionamientos. Los cuales estuvieron en buena medida motivados por la persistencia de la desocupación y la cronicidad que asumía en el caso de ciertos grupos de trabajadores. ${ }^{1}$ Pero ciertas propuestas alternativas al enfoque de la seguridad social también responden a una perspectiva valórica diferente a la implícita en aquel arreglo al plantear que debe brindarse un amplio rango de "libertad efectiva" a los ciudadanos, incluso respecto a la decisión de trabajar (para el mercado) y con qué intensidad hacerlo. A partir de allí se identifican diferentes enfoques, pero uno que cabe destacar es el del ingreso "garantido" o "básico", ${ }^{2}$ transferencia irrestricta que debería recibir toda persona independientemente de cualquier otro criterio, incluso la situación laboral.

En América Latina la brecha de pleno empleo formal es extremadamente amplia y se expresa, preferentemente, en una alta incidencia de las ocupaciones

\footnotetext{
También por otros factores como los problemas de sostenibilidad fiscal.

2 Véase, por ejemplo, Van Parijs (1992).
} 
"informales", no registradas en la seguridad social, en el sentido que no están cubiertas por ésta. En este contexto, resulta indudable que los esfuerzos por asegurar la plena vigencia de los derechos económicos y sociales no pueden recaer exclusivamente sobre la seguridad social y las regulaciones laborales.

El trabajo abogará a favor de la conveniencia de instrumentar una estrategia que se base, por un lado, en políticas destinadas a la consecución del pleno empleo formal y a consolidar un esquema de regulaciones laborales protectoras. Por el otro, en un conjunto articulado de diversas políticas sociales que garanticen el acceso universal a servicios básicos de calidad homogénea y niveles de ingresos adecuados a lo largo del ciclo de vida. Cabe reiterar que el documento busca exclusivamente contribuir a la discusión sobre un marco de referencia de las políticas sociales y laborales en América Latina, sin pretender servir de guía para el diseño y puesta en marcha de los programas específicos consistentes con esta propuesta general. Esto último requerirá de la consideración de especificidades propias de cada país en términos no sólo de las restricciones de varios tipos (institucionales, fiscales o laborales, por ejemplo) sino, también, de la arquitectura actual de las políticas públicas. Además, todo esfuerzo en la dirección de reformar las políticas sociales es básicamente un ejercicio de economía política, lo cual lleva a la necesidad de reconocer el papel de los diferentes actores sociales. En este sentido, no es posible avanzar aquí hacia mayores detalles sobre los caminos específicos a seguir -propios de cada país- ni sobre la cuantificación de los costos asociados a las reformas.

El objetivo de universalidad que aquí se plantea no significa la búsqueda de niveles mínimos de satisfacción de los derechos económicos y sociales básicos, sino el de avanzar hacia un alto grado de homogeneidad en ella. Las políticas a proponer no resultan, entonces, meros componentes de una safety net. Por su parte, el esquema aquí planteado se acerca a las propuestas tipo ingreso garantido en tanto considera transferencias no ligadas a la situación laboral, pero se diferencia radicalmente al plantear que ello no constituye el núcleo del esquema de protección y asigna relevancia a la generación de empleos de calidad así como al acceso universal de bienes y servicios como la salud, la educación, y otros que no deberían asegurarse exclusivamente por medio del mercado.

El resto del documento avanza en la discusión de la propuesta de un esquema de protección social para América Latina. En la sección 1 se discuten los límites de los sistemas de protección basados en el empleo formal mientras que en la siguiente se enuncian los componentes básicos de la estrategia de protección que parece relevante para América Latina. En la sección 3 se avanza en la descripción de los pilares de tal sistema con vocación universal pero con eje en el mercado de trabajo. En la sección 4 se discuten diversos argumentos 
en relación al papel de las regulaciones laborales en contextos de universalización de la protección social. Por último, las reflexiones. ${ }^{3}$

\section{LOS LÍMITES DE LA PROTECCIÓN SOCIAL BASADA EN EL EMPLEO EN UN CONTEXTO DE ELEVADA INFORMALIDAD EN AMÉRICA LATINA}

Los regímenes de protección social de América Latina son fundamentalmente de tipo biskmarkniano, basados en la lógica contributiva. Desde la consolidación de los rasgos básicos que actualmente caracterizan tales regímenes en América Latina, los trabajadores registrados constituyen, por tradición, una proporción reducida del empleo, significativamente menor que en los países industrializados que adoptaron ese modelo de protección.

La persistencia de una elevada informalidad en la estructura del empleo refleja que aun durante las fases de crecimiento no se ha podido avanzar significativamente hacia la reducción del que aparece como el determinante principal de aquélla, y que es una estructura productiva con una elevada participación de unidades pequeñas, de baja productividad, entre las cuales resulta más frecuente, y factible, el encubrimiento de relaciones laborales. El incremento en la informalidad durante la década de los noventa -cuando varios países flexibilizaron sus regulaciones laborales- pone al menos en entredicho el argumento según el cual sería lo estricto de estas normas una de las causas de su permanencia.

En cambio, en los 2000 se produjo una mejora asociada a un proceso de crecimiento más sostenido y más trabajo intensivo. Sin embargo, después de estos avances, la informalidad continúa caracterizando a estas economías.

El trabajo informal afecta en mayor medida a trabajadores de reducidas calificaciones y que reciben regularmente bajas remuneraciones. Precisamente, los hogares que se ubican en la parte inferior de la distribución están compuestos fundamentalmente por trabajadores de este tipo. ${ }^{4}$ Ello obedece, en parte, a

3 En este documento no se abordará la temática de la educación o vivienda, cuyo cumplimiento no estuvo tradicionalmente ligado con mecanismos de seguridad social.

4 Por ejemplo, mientras que, como promedio simple de 18 países de América Latina, 28\% de los asalariados de hogares del quintil más pobre de la distribución del ingreso familiar per cápita son formales, este porcentaje crece $76 \%$ entre los asalariados del quintil más rico (estimaciones propias sobre la base de datos de las encuestas de hogares de los países). 
la existencia de una penalidad asociada a este tipo de ocupaciones. ${ }^{5}$ La falta de cobertura resulta, asimismo, más intensa entre hogares de mayor tamaño, con mayor cantidad de nińos y con escasa capacidad de ahorro y de recursos para hacer frente a eventos laborales o de salud.

Para algunos de los riesgos que cubren los sistemas contributivos no hay (dada su naturaleza) la posibilidad de adquirir seguros en el mercado, por ejemplo, el riesgo frente al desempleo. Por otra parte, los individuos en las etapas iniciales del ciclo de vida, no tienden a ahorrar voluntariamente una parte de sus ingresos que sustente sus requerimientos durante la vejez. Asimismo, en el caso de salud la demanda de aseguramiento privado suele ser limitada entre aquellos que obtienen bajos ingresos.

En muchos países los sistemas de seguridad social son financiados por los trabajadores, los empresarios y también por el Estado. Sin embargo, los recursos fiscales suelen tener que contribuir a enjugar los déficit incluso en casos donde los trabajadores y empleadores son los únicos sostenedores del sistema. Pero aun cuando los asalariados formales terminen finalmente "pagando" por esos seguros con una remuneración neta menor a aquella que reciben si no existiesen tales costos no salariales, el resultado sería que: ahorrarían más para la vejez, demandarían más servicios de salud y tendrían mayor protección ante ciertos eventos que los informales, aun si estos últimos tuviesen un salario neto mayor que el de los formales (por la diferencia de la traslación de los costos no salariales), fenómeno que como se señaló más arriba, no se verifica en la región.

Como ya se ha manifestado, la significativa presencia de la informalidad refuerza la desigual distribución de los ingresos monetarios a lo largo del ciclo de vida. Muchas personas que participaron en el mercado laboral durante sus edades activas no logran trabajar una cantidad de años suficientes en ocupaciones registradas como para ser elegibles para el beneficio jubilatorio una vez retirados. Consecuentemente, la proporción de adultos mayores que reciben pensiones contributivas es bajo para la mayoría de los países (Rofman y Oliveri, 2011: 26-27). Por otro lado, son los adultos mayores menos educados -aquellos más propensos a trabajar en la informalidad- quienes registran una menor cobertura jubilatoria (Rofman y Oliveri, 2011: 33). Sólo en algunos

5 Estimaciones de funciones de Mincer para asalariados realizadas por los autores para Argentina, Brasil, Chile, México y Perú con datos de las encuestas de hogares cercanas a 2008 muestran que la brecha de ingresos asociada a la informalidad varía entre 0.278 para Brasil y 0.524 para Argentina. 
países como Argentina, Brasil o Costa Rica, que tienen una cobertura agregada global, no se presentan diferencias significativas entre grupos educativos. $^{6}$

Los estados han provisto tradicionalmente servicios de salud con vocación universal, gratuitamente o a bajo precio, para cubrir a quienes no son elegibles para el sistema contributivo. Ello ha facilitado un mayor acceso pero en muchos casos las prestaciones públicas (o públicamente financiadas) enfrentan limitaciones cuantitativas de oferta y/o calidad. Asimismo, el hecho de no estar cubierto por algún tipo de aseguramiento -incluida la seguridad social- está asociado a un menor uso de los servicios como muestran datos disponibles para Argentina. ${ }^{7}$

En definitiva, los ocupados informales no sólo están expuestos a una más baja estabilidad laboral ${ }^{8}$ y, por tanto, de ingresos (Beccaria y Groisman, 2006; Albornoz y Menéndez, 2002; Fields et al., 2006) y a remuneraciones inferiores, sino que tienen menos acceso a beneficios sociales de diferente tipo y no se encuentran asegurados contra eventos como los accidentes y las enfermedades laborales.

Cabe resaltar, para concluir la sección, que incluso si se llegaran a verificar condiciones favorables en términos de crecimiento económico, el proceso que llevaría a la modificación de los condicionantes estructurales de la elevada informalidad sería prolongado en el tiempo. Resulta entonces conveniente encarar de manera rápida políticas destinadas a atender la falta de cobertura que genera un sistema de protección basado fundamentalmente en una lógica contributiva y poner en marcha y/o expandir y/o consolidar otros mecanismos que operen con un enfoque universal. En las próximas secciones se plantearán caminos que se consideran adecuados para el logro de este objetivo, el cual, por otro lado, está ganando creciente consenso en la región.

6 Véase la sección 2.

7 Por ejemplo, datos de la Encuesta de Condiciones de Vida de 2001 de Argentina muestran que para el conjunto de hogares que padecieron malestares y no consultaron al médico, la proporción de aquellos que no lo hicieron por falta de dinero era mucho menor entre los no afiliados a la seguridad social, o que no contaban con un seguro privado, que entre quienes se atienden en el sector público (http://www.trabajo.gov.ar/left/estadisticas/otia/estadisticas/ verFuente.asp?Fuente=ECV.01)

8 Beccaria y Maurizio (2003), Kugler (2000) y Paes de Barros y Leite Corseuil (1999), entre otros estudios. 


\section{LOS COMPONENTES BÁSICOS DE UN ESQUEMA DE PROTECCIÓN SOCIAL PARA AMÉRICA LATINA}

El esqueleto de lo que consideramos una estrategia de protección relevante para América Latina contiene componentes de políticas contributivas, no contributivas y regulaciones laborales. El segundo de estos elementos resulta necesario ante las limitaciones de la seguridad social tradicional en mercados laborales heterogéneos. Pero también porque aparece como la forma adecuada para asegurar de manera equitativa el efectivo cumplimiento de un conjunto extenso de derechos económicos y sociales.

Por lo tanto, el esquema incluye: $i$ ) la vigencia de esquemas de seguridad social en ciertos ámbitos, como en el caso de pensiones y otros, tal como se menciona más abajo; ii) políticas de diferentes tipos - productivas, financieras, fiscales, cambiarias, comerciales, entre otras- que apunten a la promoción del empleo formal; iii) la consolidación de un conjunto de regulaciones laborales protectoras, medios para alcanzar ingresos y condiciones laborales apropiadas y para facilitar la integración social; iv) instituciones que aseguren el acceso universal a servicios de salud y educación de calidad homogénea así como niveles de ingresos adecuados, incluso durante eventos como el desempleo y luego del retiro de la vida activa.

Más allá que los mecanismos orientados a alcanzar la cobertura universal pasarían a constituirse en elementos primordiales del esquema de protección, las instituciones ligadas al empleo formal deberían continuar siendo un componente central. En este sentido, parece poco realista plantearse un camino hacia la consolidación de éste u otro sistema de protección aislado de los esfuerzos por acelerar la generación de empleo de calidad y disminuir la desigualdad de la distribución primaria del ingreso.

De manera más específica, el esquema está orientado a que toda la población acceda de manera permanente a un nivel adecuado de bienes y servicios y apuntar a reducir las desigualdades en lo que se refiere a este tema. Para ello, los individuos tendrían garantizado, por fuera del mercado de trabajo, el acceso a determinados bienes y servicios de calidad. Adicionalmente, se protegería a los individuos durante su vida activa de ciertos eventos en el plano laboral procurándose que ello ocurra independientemente de las características del puesto, especialmente de su grado de formalidad. En ese periodo se proveerían asignaciones monetarias no ligadas a su situación laboral. A su vez, se asegurarían ingresos monetarios adecuados a todos aquellos en edad de jubilación sin que se tenga en cuenta su historia ocupacional previa. 
Salud y educación constituyen los típicos ámbitos donde los esfuerzos deberían encaminarse a cubrir a toda la población con servicios de elevada y homogénea calidad, y que no se vincule a los niveles de recursos de los hogares. Esto es, la seguridad social no parece un esquema apropiado para el primero de esos ámbitos.

Las regulaciones del mercado de trabajo continuarán protegiendo a los asalariados formales, con escasas posibilidades de que puedan extenderse al resto de los trabajadores. Uno de los riesgos cuya cobertura debería procurar ser extendida al conjunto de los ocupados es el desempleo, aspecto sobre el cual se volverá más adelante.

Las transferencias monetarias contribuirían a lograr niveles adecuados de ingresos a lo largo del ciclo de vida. Entre los que alcanzan la edad legal de retiro, los montos deberían asegurar un acceso adecuado a los bienes y servicios que se obtienen en el mercado, protegiendo entonces a quienes no reciben jubilaciones o pensiones de la seguridad social, o a los que lo hacen en montos muy bajos.

Convendría recalcar nuevamente que el esquema de protección que aquí se esboza resulta de una articulación de los mecanismos de seguridad social con una pluralidad de programas y políticas que en conjunto apuntarían a asegurar la cobertura universal de los derechos económicos y sociales. Difícilmente en muchas áreas pueda considerarse un único dispositivo que permita alcanzar ese objetivo. Los componentes específicos y la importancia relativa que adquieran las diversas instituciones dependerán de la realidad de cada país y serán seguramente cambiantes en el tiempo. Esta necesaria combinación de varias acciones obedece a dos razones, por un lado, a que cuestiones de eficiencia, y también de realismo político, llevarán a que en ciertas áreas se mantengan instrumentos de la seguridad social, los que se combinarían con programas no contributivos. Por el otro, a que el objetivo universalista requerirá seguramente de instrumentos que focalicen determinadas acciones o prestaciones en ciertos grupos, precisamente los más desventajados y que requieran de atenciones especiales.

\section{ALGUNOS RASGOS DE LA ARQUITECTURA DE LOS INSTRUMENTOS DE PROTECCIÓN CON ORIENTACIÓN UNIVERSAL}

En esta sección se discutirán los rasgos principales de algunas políticas, instituciones o programas en los cuales se expresa el sistema de protección social cuyos objetivos y trazos básicos ya fueron planteados. No se abordarán las regulaciones laborales mientras que en la sección siguiente se analizarán en 
particular las relacionadas con la estabilidad en el empleo y el salario mínimo en el marco de una discusión más amplia respecto de la relación entre un enfoque de protección social universal y las normas laborales.

Cabe reiterar que la instrumentación del esquema, a partir del despliegue de ciertos dispositivos y/o la adecuación de otros, sólo podría efectuarse de manera paulatina, fundamentalmente, por la necesidad de afianzar un proyecto político que lo haga suyo; pero también, porque deben superarse restricciones de tipo financieras, institucionales o las derivadas de la resistencia de sectores económicos y/o sociales.

El examen de algunos componentes que aquí se efectuará sólo pretende contribuir a aclarar aspectos del esquema global, por lo que no abordará cuestiones de detalle. En particular, se explayará en mayor medida sobre algunos casos en los cuales se considera que la universalización de la protección debería implicar la articulación de instrumentos de la seguridad social con otros que atiendan a los que están excluidos de su alcance. Si bien se busca avanzar hacia el logro de la mayor homogeneidad posible en la protección, en ciertas circunstancias parece más conveniente -como ha sido mencionado- contar con instituciones (y niveles de beneficio) algo distintas entre esos dos grupos de individuos. Ello no debería, sin embargo, derivar en una segmentación marcada entre la protección que obtienen unos y otros.

La forma de asegurar prestaciones universales y relativamente homogéneas es creando un sistema nacional que cubra a todos los individuos, independientemente de la situación laboral, la edad o el género. Avanzar en esta dirección enfrenta obvias dificultades de financiamiento y también de "economía política”, en tanto quienes se atienden en subsistemas contributivos -que brindan prestaciones adecuadas a bajos costos- tenderán a resistir la integración de los mismos con otros componentes, especialmente el público.

Una etapa hacia la meta de mayor equidad sería el mejoramiento de la calidad de los servicios prestados a aquellos sin cobertura de seguridad social ni privada, lo cual requerirá de un incremento del gasto público en salud. Algunos países de América Latina han dado algunos pasos en esta dirección mediante mecanismos, y con alcances, diferentes. Ya en 1993 Colombia introdujo una reforma encaminada al aseguramiento universal ${ }^{9}$ en tanto apunta a establecer el acceso de toda la población a un paquete básico de prestaciones. A partir de la misma, aquellos no cubiertos por la seguridad social (excluyendo miembros de hogares de altos ingresos) pueden inscribirse en aseguradoras que 
reciben una capitación por parte del Estado para financiar al plan obligatorio de atención (Giedion y Acosta, 2011: 32-33). En Argentina hay un programa que incentiva a los prestadores del sector público a elevar la cobertura efectiva -y calidad de servicios- en la atención de salud materno-infantil. Similar objetivo -y lógica de funcionamiento- tiene el programa de maternidad gratuita de Ecuador. En Chile se encuentra en pleno funcionamiento un programa que asegura la atención oportuna a cualquier persona con un conjunto amplio de patologías. Si bien hay un copago por parte del beneficiario, el mismo depende del nivel de recursos (no lo pagan aquellos de muy bajos ingresos) y se estipula un máximo anual en término de cantidad de remuneraciones mensuales (Drago, 2006: 37).

Estos son esfuerzos de ampliación de la cobertura efectiva y mejora de la calidad de los servicios sanitarios pero su efecto sobre la desigualdad global en términos de atención y resultados es aún limitado. Prevalece, además, la segmentación del sistema; así, por ejemplo, en Colombia, donde si bien las aseguradoras del componente subsidiado pueden contratar los mismos efectores que atienden a los otros subsistemas, no se plantea una integración más completa de los mismos (Giedión y Acosta, 2011: 139-141).

Costa Rica ha contado tradicionalmente con un sistema que brinda servicios homogéneos por medio del organismo de seguridad social -la Caja Costarricense de Seguridad Social (CCss) - a una amplia población (86\%). Están afiliados al seguro médico no sólo los asalariados registrados y los trabajadores por cuenta propia formales sino también los pensionados y jubilados del régimen contributivo y no contributivo. Desde 1984 el Estado además financia la afiliación de miembros de hogares de bajos ingresos. Hacia esa dirección se movió también Brasil a partir de la reforma constitucional de 1998 sobre la que se basó la creación del Sistema Único de Salud (sus), que cubre a todas las personas, sean a no contribuyentes a la seguridad social. Este proceso ha facilitado la efectiva integración y racionalización del sistema así como avances hacia una mayor equidad. Sin embargo, también se advierte la persistencia de diferencias en la calidad con los servicios provistos y financiados privadamente. Esto provoca que la mayoría de la población combine la atención en el sus con esos seguros privados o pagos directos (Becerril Montekio et al., 2011: 124-125).

Diferentes pueden ser, entonces, las formas para avanzar hacia la universalización de la salud, no sólo de la cobertura sino también en términos de reducción de disparidades en la calidad de la atención. Algunos procesos que elevan efectivamente el acceso y mejoran las prestaciones que reciben los hogares de menores recursos pueden, sin embargo, cristalizar diferencias entre 
componentes del sistema sanitario; otros, en cambio, tienen un diseño que procura una mayor integración y menor segmentación. En particular, estos últimos requieren de estrategias que consideren la elevación del grado de coordinación entre los subsistemas y permitan un uso eficiente de los recursos disponibles.

A fin de contribuir con la equidad horizontal -y que, en la práctica, implica equidad vertical- un seguro de desempleo de naturaleza contributiva debería ir acompañado, como un elemento central del sistema de protección, de un mecanismo que proteja a los individuos ante el evento de desempleo proveniente de la pérdida de un puesto informal. ${ }^{10}$ Este último sería un instrumento que transfiriera ingresos a los que experimentan tal situación y que proveyera similares incentivos que el seguro contributivo. Pero por las diversas razones que se comentarán inmediatamente, no sería posible adecuar estos seguros para que cubran a los trabajadores informales, por lo que debería tenerse en cuenta un dispositivo específico.

Un aspecto crucial que debe considerarse en su diseño, es la manera de identificar la condición de desocupado. Una alternativa sería requerir que el beneficiario realice alguna actividad durante el periodo que está cubierto (capacitación u ocupación) y que revele aquella condición.

Pero dadas las características mismas de los mercados laborales de América Latina, no hay una plena diferenciación o discontinuidad entre la desocupación abierta y el empleo en ciertos puestos de unidades no estructuradas, informales. Estos últimos resultan frecuentemente refugios frente al desempleo abierto, generando muy bajos ingresos que, en muchos casos, tampoco les asegura el acceso a los bienes y servicios básicos. Por tanto, no parece posible restringir el programa a los desocupados abiertos no cubiertos por el sistema contributivo ya que resultará, asimismo, atractivo para muchos que trabajan en una ocupación informal y obtienen ingresos muy bajos; por tanto, estos últimos deberían ser también elegibles para este dispositivo. Un programa de este tipo estaría cumpliendo el objetivo de asegurar un determinado nivel de ingreso a todos los que deseen trabajar y no encuentren una ocupación que les permita lograr ese nivel. En tanto la cantidad de solicitantes resulte amplia, este objetivo difícilmente se cumplirá plenamente con un mecanismo como el comentado, entre otras cosas, por las dificultades que pueden preverse rela-

10

Incluso para los trabajadores formales la preocupación por la atención al desempleo no ha sido prioritaria en América Latina: sólo seis países cuentan con seguros contributivos y en esos países, la cobertura es reducida (entre 10 y $20 \%$ de los desocupados). Véase, por ejemplo, Velásquez Pinto (2010: 11). 
tivas a su gestión. En ese sentido, podría complementarse con programas de generación directa de empleo. En realidad, si el requisito que se impone a las personas que solicitan el beneficio para exteriorizar su condición de desocupado es el desempeño de un puesto de trabajo, el dispositivo no se diferencia sustancialmente de un programa activo de empleo.

En cuanto al monto del beneficio, debería ser algo menor que el mínimo del seguro contributivo para no afectar ciertas decisiones de oferta. Su duración, por otra parte, no podría ser muy corta o, más estrictamente, establecida bajo la consideración que la desocupación y la subocupación asociada a la informalidad sean fenómenos coyunturales o friccionales. La naturaleza estructural de la subocupación en América Latina requeriría de una protección más prolongada.

Tanto la elevada "demanda" potencial de estos programas, como la necesidad de que su duración no resulte corta, implicarían una carga muy significativa sobre los recursos fiscales. Por lo tanto, su instrumentación también resultaría necesariamente progresiva. El monto de la asignación constituirá un parámetro básico ya que la auto-focalización llevará a que cuanto menor sea éste, más reducida será la proporción de ocupados en puestos informales que solicitarían ingresar al sistema. También podrían considerarse criterios de entrada -tomando en cuenta ciertas características individuales o del hogar-y relajarlos de manera progresiva.

Son escasas las experiencias de atención del riesgo de desempleo para los trabajadores informales. Una de las que ha intentado enfrentar tal situación en Argentina es el Seguro de Capacitación y Empleo (SCE). Sus beneficiarios reciben una transferencia monetaria durante un máximo de dos años y deben realizar actividades de capacitación mientras permanecen en el programa. El monto de la transferencia es, sin embargo, muy reducido (10\% del salario mínimo en 2012), hecho en parte relacionado con el bajo monto del seguro contributivo. ${ }^{11}$ Si bien el programa está destinado a los desocupados no elegibles para el seguro contributivo, en la práctica la mayor parte de quienes lo reciben eran beneficiarios de otros programas sociales que están siendo eliminados o reducidos. Otros programas que brindan capacitación a personas desempleadas o subempleadas lo tienen en México, Chile y Uruguay, de duraciones más acotadas y/o destinados a trabajadores de ciertas actividades.

La generación directa de empleo por parte del Estado, y otras políticas activas como las de promoción del autoempleo, también pueden considerarse

11 El monto máximo del seguro de desempleo equivale a 19\% del salario mínimo. 
como mecanismos para atender situaciones de desocupación o subocupación. Ejemplo de ello son el Programa de Apoyo a la Inserción Laboral en Panamá, el Programa de Apoyo a Emprendimientos Productivos en Uruguay o el Programa Nacional de Emprendedores en México.

Para alcanzar el objetivo de asegurar niveles de ingresos adecuados a todos los adultos mayores deberían establecerse dispositivos que cubran a aquellos no elegibles para el régimen contributivo. Parece, por tanto, conveniente estructurar un sistema de pensiones con varios componentes adicionales al contributivo. Uno de estos nuevos pilares brindaría una prestación asistencial a quienes no hayan realizado ningún aporte a la seguridad social. En otro, serían elegibles quienes lo hayan hecho durante una cantidad de tiempo que no le permite alcanzar un haber mínimo a definir. Esos últimos recibirían una prestación asistencial pero se les reconocería, adicionalmente, un monto que se calcularía en función de los aportes realizados.

En un esquema de este tipo, el pilar contributivo continuaría siendo el principal, con lo cual se mantendrían cierto incentivo al trabajo y a la registración. De cualquier manera la diferencia de las prestaciones entre ambos componentes no debería ser muy amplia ya que la no elegibilidad para el contributivo resulta en muchos casos de trayectorias laborales que implicaron movimientos básicamente involuntarios. Nuevamente por razones fiscales, un cambio en esta dirección procederá generalmente de manera progresiva.

La preocupación por extender algún tipo de beneficio monetario a los adultos mayores no elegibles para el régimen contributivo en América Latina es, salvo en el caso de Brasil, relativamente reciente. Ello se ha hecho en la mayoría de los casos por medio, precisamente, de la creación de pilares no contributivos. Así, en Chile, hacia 2008, se estableció un esquema ${ }^{12}$ que fue cubriendo progresivamente al segmento de esa población que pertenece al $60 \%$ más pobre. Como resultado, aproximadamente la mitad de los mayores de 64 años que no recibe pensiones contributivas pasó a ser beneficiario de algún esquema no contributivo (Rofman et. al., 2010: 98). Como consecuencia de la introducción de un amplio programa de naturaleza no contributiva, la proporción de la población adulta mayor que recibió algún beneficio previsional pasó de $20 \%$ a mediados de los noventa a $90 \%$ hacia finales de los 2000 . También en Ecuador se introdujo, en 1998, un esquema destinado a atender a quienes no eran elegibles para el régimen contributivo (cerca de $80 \%$ de ellos

12 Que remplazó a otros también de ese carácter y que incluso garantizaba una pensión mínima a quienes aportaron durante 20 ańos pero acumularon un monto inferior al necesario para ser elegibles. 
mayores de 64 años) llevando la cobertura total a 50\%. Costa Rica cuenta, asimismo, con un régimen no contributivo. Brasil había sido pionero en estos esfuerzos ya que a finales de los ochenta introdujo las pensiones rurales, que beneficiaron a un segmento particularmente ausente de la seguridad social.

Argentina no creó otros pilares pero redujo los requerimientos de acceso al régimen contributivo. El Plan de Inclusión Previsional permitió que las personas que se encontraban en edad jubilatoria y no habían realizado aportes, o les faltaban años por hacerlos, ingresasen a una moratoria con lo que accedieron a la jubilación. La tasa de cobertura de los adultos mayores aumentó del mínimo histórico de alrededor de 67\% en 2004 a 90\% en 2009 (Curcio y Beccaria, 2011: 86-88). En Uruguay también se relajaron las condiciones de entrada al sistema contributivo al reducir la cantidad mínima de años de aporte (de 35 a 30), aunque además se amplió el programa no contributivo. ${ }^{13}$

Sin embargo, fuera de estos países, ${ }^{14} \mathrm{y}$ en otros como Panamá, la cobertura en el resto de América Latina no supera aún 30\% y se ubica en alrededor de $20 \% .^{15}$

En resumen, el esquema hasta ahora descrito garantizaría un ingreso monetario mínimo a todas las personas u hogares a partir de un sistema jubilatorio con pilares contributivos y no contributivos, el salario mínimo y el seguro de desempleo para los trabajadores formales, y una transferencia a los desocupados y trabajadores informales de bajos ingresos. Este complejo de dispositivos, sin embargo, deja sin proveer daciones monetarias a las personas en edad activa que sin tener un impedimento físico no deseen trabajar. Como tal situación podría obedecer a que estos individuos realicen tareas de cuidado y/o comunitarias, se propone también un subsidio básico universal a todas las personas u hogares. Pero la conveniencia de considerar esta transferencia como parte del esquema también radica en su papel como atenuador de las brechas de ingresos monetarios, entre los que se encuentran recibiendo los beneficios monetarios mínimos y los insertos de manera más satisfactoria en el mercado de trabajo o gozan de pensiones contributivas. Su monto no pretende cubrir las necesidades básicas, los que sólo se atenderían con el agregado de esta transferencia a los seguros de desempleo o al salario mínimo.

13 En 2008 se estableció un beneficio no contributivo para las personas entre 65 y 70 años en situación de pobreza que no accedían al sistema contributivo. Véase Bertranou et al. (2011:40).

14 La "Pensión básica universal" de El Salvador y la "Pensión 65" de Perú constituyen también pilares no contributivos, pero su cobertura es todavía limitada (CAF, 2012: 76).

15 Los párrafos anteriores sobre las experiencias de programas no contributivos en América Latina se basan en Rofman y Oliveri (2011: 26-35). 
Dadas las dificultades que pueden preverse en el proceso de ampliación de la cobertura de los beneficios arriba comentados para los ocupados y desocupados informales y para los inactivos, quizás convendría avanzar hacia el objetivo de asegurar un ingreso mínimo a los individuos en edades activas ( $\mathrm{y}$ a sus hogares) por medio de una combinación de esos dispositivos con una transferencia universal. Se está haciendo referencia a esquemas similares a los instrumentados recientemente en Argentina y Uruguay que extiende la asignación familiar a los hogares sin empleados formales con presencia de menores, aunque se considera que la transferencia debería se incondicionada, a diferencia de lo que acontece con esos programas que acaban de mencionarse. Como el aumento de la cobertura de un programa de este tipo resulta más sencillo, al menos desde el punto de vista de la gestión, se podría tener como objetivo universalizar la cobertura del mismo y avanzar de manera más paulatina con la aplicación de dispositivos no contributivos que atiendan el riesgo del desempleo.

Las transferencias monetarias focalizadas, condicionadas a la demostración de medios (means-tested), no formarían parte del esquema aquí esbozado ya que el entramado propuesto de dispositivos terminarían teniendo una cobertura universal. Sin embargo, dado precisamente lo comentado en el párrafo anterior y lo señalado en diversos puntos de esta sección respecto a que los elementos que se están describiendo resultan un objetivo de máxima y de largo plazo, la focalización tendría un papel instrumental en un proceso que será seguramente progresivo. En efecto, algunas de esas transferencias se aplicarían de manera paulatina, considerándose criterios de focalización.

Pero, ésta -como ya se señaló- cumple también otro papel ya que de manera sistemática, o al menos durante periodos que pueden ser prolongados, la efectiva homogeneidad en el acceso y calidad de los satisfactores (y en los resultados logrados) requerirá de políticas que atiendan especialmente a determinados grupos que vienen enfrentando las mayores dificultades. Por ejemplo, el tipo de apoyo requerido para asegurar la permanencia escolar y las mejoras en la calidad debería ser más intenso para ciertos segmentos de la población que para otros grupos.

\section{PROTECCIÓN SOCIAL UNIVERSAL Y REGULACIONES LABORALES}

En esta sección se aborda una temática que permitirá otorgarle mayor claridad a la propuesta, como son los posibles conflictos entre, por un lado, un esquema de protección que aspira a asegurar a todos los ciudadanos el pleno disfrute de sus derechos económicos y sociales y, por el otro, un entramado denso de regulaciones del mercado de trabajo. Si la garantía de los derechos no depende 
en última instancia de la condición laboral de la persona, ¿cuál sería la necesidad de promover la creación de empleo y, más importante aún, de empleo protegido por diversas regulaciones que aseguren, entre otros objetivos, niveles adecuados de estabilidad laboral y remuneraciones mínimas? Podría argumentarse que tener los derechos garantizados por fuera del mercado de trabajo brindaría una mayor libertad al individuo y le permitiría ser (más) selectivo en el tipo de empleo que acepte; esto es, le eleva el salario de reserva. De la misma manera, el perjuicio de la inestabilidad laboral se morigera al brindarse cierto aseguramiento al ingreso. Consecuentemente, algunas normas laborales aparecerían como redundantes por lo que podrían eliminarse o flexibilizarse, lo cual llevaría a ganancias de eficiencia y crecimiento del empleo; este sería el caso, en particular, de las concernientes a la seguridad en el empleo y el salario mínimo.

En efecto, se ha planteado frecuentemente que la primera de esas dos instituciones provoca desaliento a la contratación, limita la introducción de tecnología y debilita la movilidad en respuesta a cambios en la distribución sectorial y/o regional de la producción. Pero las dificultades serían aún mayores porque el sistema de beneficios crecientes según la antigüedad no guarda relación con el riesgo de desempleo asociado a la fase del ciclo económico (Velásquez Pinto, 2010). Esto último también desincentivaría el despido de trabajadores de mayor antigüedad y, por ende, haría recaer el costo del ajuste mayormente sobre los trabajadores más jóvenes. La rotación laboral, a su vez, permitiría aumentos de productividad debido a la difusión de saberes y a la mejor asignación del factor trabajo y la desocupación, como estado que facilita la búsqueda de empleo, conduciría a una movilidad ascendente y a una mayor eficiencia asignativa. En resumen, el argumento central a favor de sistemas de protección que descansan en un ingreso universal garantizado y/o la universalización del acceso a determinados servicios básicos, es el efecto favorable de la disminución del costo laboral sobre la eficiencia asignativa y la generación de empleo.

Sin embargo, estos argumentos están lejos de ser constatados suficientemente por la evidencia empírica disponible y hay una amplia disputa acerca de los efectos de las experiencias flexibilizadoras sobre los niveles y calidad del empleo. En América Latina, desde comienzos de la década de los noventa (y en algunos casos con anterioridad) se produjeron reformas desregularizadoras que disminuyeron los costos laborales no salariales y sin lograr mejoras en el mercado de trabajo (Marshall, 1996; o Weller, 2001).

Pero más allá de la evaluación de aquellas experiencias, se desea enfatizar que la disminución de los costos laborales no debería ser el objetivo central que persigan las políticas tendientes a la universalización de la protección. Esta orientación está enraizada en principios básicos, como el de los derechos de los 
ciudadanos que plantea que el ejercicio pleno de los mismos requiere el acceso a determinados recursos básicos. Los ciudadanos son titulares de derechos y la sociedad debe establecer mecanismos que permitan que ellos sean efectivamente garantizados.

En este contexto, el diseño de las regulaciones laborales debe responder a los méritos relativos de sus diferentes componentes en lo que hace a aspectos como el bienestar de los trabajadores. Este diseño no resulta, por cierto, independiente del que corresponde a los mecanismos de protección, como los de salud y previsión. Sin servicios sanitarios y de pensiones con vocación universalista las propuestas de flexiseguridad, por ejemplo, podrían afectar la calidad de vida de los trabajadores. Sin embargo, el balance entre los componentes que promueven la estabilidad en el empleo y los que proveen seguridad del ingreso debería evaluarse en términos de los objetivos y efectos de cada uno de ellos. Si bien el desincentivo al despido, que buscan los primeros, eleva la estabilidad del flujo de remuneraciones, objetivo que es similar al de los seguros de desempleo o a un ingreso básico, las normas sobre seguridad en el empleo apuntan a un conjunto de metas más amplio, tanto en el plano del bienestar como en el de la eficiencia productiva.

Así, la pérdida de un empleo puede acarrear la ruptura de lazos importantes de sociabilidad generados en el mundo del trabajo. Tampoco se enfatizan las ventajas de las relaciones laborales de largo plazo sobre la eficiencia, en tanto resultan más propicias para la capacitación en el puesto de trabajo que las más efímeras y, además, promueven una relación más cooperativa entre trabajadores y empleadores. Si bien las empresas podrían decidir per se una mayor estabilidad, la existencia de costos o limitaciones al despido constituyen incentivos dirigidos a influenciar el comportamiento de las firmas en la dirección de promover relaciones laborales más estables.

Una transferencia que garantice un nivel mínimo de ingresos a los hogares también suele ser vista como un sustituto del salario mínimo. La opción más eficiente es que el mercado fije las remuneraciones y se alcance así el pleno empleo, objetivo que no se lograría de estar vigente aquella institución laboral; su influencia también provocaría una sustitución de ciertos tipos de trabajadores por otros; por ejemplo, de no calificados por calificados, de jóvenes por adultos y de mujeres por varones, dado que es más probable que los salarios de los primeros sean iguales o cercanos al salario mínimo. Si el salario de equilibrio que se fija el mercado resulta insuficiente para garantizar un nivel de vida adecuado al hogar del trabajador, la brecha sería cubierta por la transferencia.

Sin embargo, por un lado, hay esquemas conceptuales alternativos, como el modelo de mercados monopsónicos, a partir del cual un aumento del valor 
del mínimo no necesariamente implicará reducciones en el empleo, pudiéndose observar un efecto neutro o incluso un incremento (Manning, 2003: 3-28), por otro lado, las investigaciones para la región muestran, en general, un bajo o nulo efecto negativo del salario mínimo sobre los niveles de empleo agregado. ${ }^{16}$

Asimismo, los incrementos en los mínimos podrían promover cambios en la organización del trabajo que deriven en ganancias de productividad. Desde el lado de la demanda, aumentos salariales a trabajadores con baja propensión a ahorrar pueden generar un incremento en el consumo interno con efectos positivos sobre la creación global de empleo.

Si bien, entonces, es razonable evaluar la relación entre salario mínimo y nivel de empleo, en los países en desarrollo que cuentan con una amplia oferta excedente de trabajo (no sólo reflejada en el desempleo abierto) no resultaría adecuado eliminar el salario mínimo o dejarlo erosionar a niveles muy reducidos, incluso por razones de eficiencia. Parece escasamente atractiva una estrategia de desarrollo que base el crecimiento del empleo en salarios bajos.

Para recapitular, se ha argumentado que los avances hacia la universalización de los derechos económicos y sociales básicos de los ciudadanos no pueden constituirse automáticamente en el justificante para eliminar o hacer más laxas las regulaciones laborales. En el marco de la aplicación de esa estrategia, las normas del mercado de trabajo pueden requerir de adecuaciones, pero no deben perderse de vista los efectos globales de estas normas, tanto en el plano de la eficiencia como en el de la equidad.

\section{REFLEXIONES FINALES}

La elevada desigualdad del bienestar que caracteriza a América Latina refleja una marcada concentración de la distribución primaria de los ingresos monetarios así como la presencia de sistemas de protección que no permiten moderar los efectos de aquella, y en muchos casos, los refuerzan. Esto obedece, en parte, a que tales sistemas responden, en buena medida, a mecanismos de seguridad social, los que naturalmente no cubren a la amplia porción de la población no ligada, o sólo débilmente ligada, al empleo formal.

16 Ni Bell (1997: 24) para México, ni Lemos (2007; 219) para Brasil, encuentran efectos significativos del salario mínimo sobre el empleo. Estos resultados difieren de los encontrados por este último autor en estudios previos donde se sugería la existencia de un efecto de largo plazo negativo, si bien de reducida magnitud (Lemos 2004: 1). Gindling y Terrell (2007: 22) estiman una elasticidad del empleo total al sm de -0.10 en el caso de Costa Rica. 
Como respuesta a esta situación, se ha abogado por cambios en los sistemas de protección que responden a diversas orientaciones como aquellos que plantean modificar completamente la arquitectura de aquellos basados en la seguridad social o los que buscan orlar a ésta con redes que atienda a grupos que enfrentan las condiciones más difíciles.

En este trabajo se han planteado los rasgos generales de un enfoque que implica avanzar hacia la cobertura universal de servicios de salud, previsión, compensación contra el desempleo y obtención de ingresos adecuados, recurriendo tanto a mecanismos contributivos como no contributivos y que, en conjunto, aseguran que toda la población goce de los derechos económicos y sociales básicos.

Se enfatiza aquí la necesidad de proceder a lo largo de un camino de incremento de la cobertura mediante diferentes instrumentos y políticas de protección social; también se subraya el papel relevante que tiene el mercado de trabajo como eje de integración social. De allí la importancia que se asigna al dinamismo del empleo decente como medio para mejorar el nivel de bienestar de la población y reducir la inequidad. Cabe tener en cuenta que las estrategias de universalización aquí propuestas no eliminarán todas las brechas que hay entre la calidad de vida de los trabajadores formales e informales. Algunas de éstas podrán ser temporales pero otras se mantendrían de manera más persistente ante la dificultad de extender completamente la cobertura de ciertos riesgos, como los de seguridad e higiene laboral, o por las brechas que continuarían presentes, por ejemplo, entre las prestaciones que obtendrían beneficiarios de los pilares contributivos y no contributivos de pensión.

Por lo tanto, el avance hacia la universalización de la protección debería ir acompañado, por un lado, de regulaciones que mantengan, y si es posible, eleven, las condiciones de trabajo de las ocupaciones formales. Por el otro, de políticas que fomenten el crecimiento de los puestos de buena calidad en términos de estabilidad, condiciones de trabajo y productividad. Las enormes diferencias que persisten en la calidad de los empleos en la región requieren que se actúe sostenidamente en la distribución primaria del ingreso como vía para reducir la inequidad y permitir que la obtención de un empleo sea un mecanismo efectivo de salida de la pobreza y una puerta de entrada a la protección.

La discusión realizada alrededor de un blue-print o esquema de protección social no pretende más que contribuir a la reflexión acerca del paradigma que podría guiar la modificación de los sistemas actualmente vigentes en América Latina. Pero todo paradigma de protección social constituye un aspecto central-explícito o implícito- de cualquier propuesta política y no sólo responde a la perspectiva ideológica y al enfoque sobre otros componentes de ese pro- 
yecto, sino a las reales posibilidades de instrumentación, las cuales se refieren, fundamentalmente, a las de naturaleza política pero también responderán a la realidad económica e institucional de cada país.

Sea cual fuere el planteamiento general que se vaya delineando, su puesta en marcha será paulatina ya que los acuerdos específicos no siempre serán fáciles de alcanzar y también porque deben irse superando restricciones, especialmente institucionales y fiscales. Por tanto, la ampliación de la protección comenzaría por los hogares más vulnerables, y apuntando a mitigar las tendencias a la transferencia intergeneracional de la pobreza.

\section{BIBLIOGRAFÍA}

Albornoz, Facundo, y Marta Menéndez (2002), Analyzing income mobility and inequality: the case of Argentina during the 1990's, París, inédito.

Beccaria, Luis, y Roxana Maurizio (2003), Movilidad ocupacional en Argenti$n a$, Los Polvorines, Universidad Nacional de General Sarmiento.

Beccaria, Luis y Fernando Groisman (2006), "Inestabilidad, movilidad y distribución del ingreso en Argentina”, Revista CEPAL, núm. 89, Chile, pp. 133-156.

Becerril Montekio, Víctor; Guadalupe Medina, y Rosana Aquino (2011), "Sistema de salud de Brasil", Salud Pública de México, vol. 53, núm. 2, México, pp. S120-S131.

Bell, Linda (1997), "The impact of minimum wages in Mexico and Colombia”, Journal of Labour Economics, vol. 15, núm. 3, pp. 103-135.

Bertranou, Fabio; Óscar Cetrángolo; Carlos Grushka, y Luis Casanova (2011), Encrucijadas en la seguridad social argentina: reformas, cobertura y desafios para el sistema de pensiones, Buenos Aires, CEPAL y Oficina Internacional del Trabajo.

Corporación Andina de Fomento (CAF) (2012), Finanzas públicas para el desarrollo, Bogotá, CAF.

Curcio, Javier, y Beccaria, Alejandra (2011), "Sistema de seguridad social y mercado de trabajo: evolución de la cobertura en la Argentina entre 1990 y 2010", en Claudia Danani, y Susana Hintze (coords.), Protecciones y desprotecciones: la seguridad social en la argentina 1990-2009, Los Polvorines, Universidad Nacional de General Sarmiento, pp. 61-101.

Drago, Marcelo (2006), La reforma al sistema de salud chileno desde la perspectiva de los derechos humanos, serie Políticas Sociales, 121, Santiago, CEPAL, pp. 1-94. 
Fields, Gary; Robert Duval Hernández; Samuel Freije Rodríguez, y María Laura Sánchez Puerta (2006), Income mobility in Latin America, Cornell University, ILR working papers collection.

Giedion, Úrsula, y Olga Acosta (2011), "Sistema de salud y democracia: el caso de Colombia”, en Alicia Bárcena y Osvaldo Kacef, La política fiscal para el afianzamiento de las democracias en América Latina, Santiago, CEPAL-PNUD, pp. 127-156.

Gindling, Thomas, y Katherine Terrell (2007), "The effects of multiple minimum wages throughout the labor market: the case of Costa Rica", Labour Economics, vol. 14, núm. 3, pp. 485-511.

Kugler, Adriana (2000), The incidence of job security regulations on labor market flexibility and compliance in Colombia: Evidence from the 1990 reform, documento de la Red de Centros de Investigación del BID, núm. R. 393.

Lemos, Sara (2004), "Minimum wage policy and employment effects: Evidence from Brazil”, Economia, vol. 5, núm. 1, pp. 219-266.

(2007), "Minimum wages across the public and private sectors in Brazil”, Journal of Development Studies, vol. 43, núm. 4, pp. 700-720.

Manning, Alan (2003), Monopsony in motion: Imperfect competition in labor markets, Princeton, Princeton University Press.

Marshall, Adriana (1996), "Protección del empleo en América Latina: las reformas de los años 1990 y sus efectos sobre el mercado de trabajo", Estudios del Trabajo, núm. 11, ASET, Buenos Aires, pp. 3-29.

Paes de Barros, Ricardo, y Carlos Leite Corseuil (1999), Labor market regulations and the duration of employment in Brazil, IPEA, working paper, núm. 676.

Rofman, Rafael; Eduardo Fajnzylber, y Germán Herrera (2010), "Reformando las reformas previsionales: en la Argentina y Chile", Revista CEPAL, núm. 101, Santiago, pp. 85-109.

Rofman, Rafael, y María Laura Oliveri (2011), La cobertura de los sistemas previsionales en América Latina: conceptos e indicadores, Buenos Aires, Banco Mundial.

Velásquez Pinto, Mario (2010), Seguros de desempleo y reformas recientes en América Latina, serie Macroeconomía del Desarrollo, núm. 99, Santiago, CEPAL.

Van Parijs, Philippe (1992), "Competing justifications of basic income", en Philippe Van Parijs (ed.), Arguing for basic income, ethical foundations for a radical reform, Nueva York, Verso, pp. 3-43.

Weller, Jurgen (2001), Economic reforms, growth and employment: labour markets in Latin America and the Caribbean, Santiago, CEPAL. 